\title{
Coulisses
}

Revue de théâtre

44 | Printemps 2012

Le Hors-scène

\section{Crise identitaire et théâtre citoyen chez Maurice Bandaman}

Une lecture de Au nom de la terre

\section{Bassidiki Kamagaté}

\section{OpenEdition}

\section{Journals}

Édition électronique

URL : https://journals.openedition.org/coulisses/478

DOI : $10.4000 /$ coulisses.478

ISSN : 2546-9460

\section{Éditeur}

Presses universitaires de Franche-Comté

Édition imprimée

Date de publication : 1 juin 2012

Pagination : 149-162

ISBN : 978-2-84867-425-4

ISSN : $1150-594 \mathrm{X}$

\section{Référence électronique}

Bassidiki Kamagaté, « Crise identitaire et théâtre citoyen chez Maurice Bandaman », Coulisses [En ligne], 44 | Printemps 2012, mis en ligne le 30 novembre 2016, consulté le 29 décembre 2022. URL http://journals.openedition.org/coulisses/478 ; DOI : https://doi.org/10.4000/coulisses.478 


\title{
Crise identitaire et théâtre citoyen chez Maurice Bandaman : une lecture de $A u$ nom de la terre
}

\author{
BASSIDIKI KAMAGATÉ \\ Université de Bouaké, Côte d'Ivoire
}

L'image de goulags tropicaux offerte par les régimes politiques africains postcoloniaux a contraint de nombreux dramaturges à faire de leurs œuvres le creuset des récriminations et des aspirations légitimes de peuples victimes de la férocité extrême d'une poignée d'individus assoiffés de pouvoir et arc-boutés à leurs privilèges : c'est l'émergence en Afrique du théâtre politique et politisé des années 1970 et 1980. Bernard Dadié avec Les Voix dans le vent ou encore Aimé Césaire dans Une saison au Congo pouvaient alors pourfendre tout ce qu'ils considéraient comme des entraves politiques à l'essor de l'Afrique. Face au désenchantement et à la grosse désillusion des indépendances, ils ne pouvaient se taire en fermant les yeux sur l'imposture dictatoriale des nouveaux dirigeants et les différents dysfonctionnements de la nouvelle société émergente. Le théâtre en devient un réceptacle des contingences socio-politiques de l'Afrique postcoloniale.

Ce paradigme idéologico-littéraire, à l'origine d'une littérature sectaire et à la limite folklorique, se voit contesté à partir des années 1990 à l'avènement de dramaturges que Sylvie Chalaye se plait à appeler «Les enfants terribles des indépendances $»^{1}$ dont les œuvres, déstigmatisées de l'Afrique, s'imprègnent de la multiculturalité et de la transculturalité, signes de leur ouverture au monde et de leur refus d'une littérature de soi sur soi. Toutefois, les prétentions universalistes de ces auteurs iconoclastes (qui vivent pour la plupart en exil) n'ont pas encore trouvé écho auprès de tous les auteurs dramatiques, notamment auprès de ceux restés sur le continent qui ne semblent pas avoir renoncé aux enjeux socio-politiques de leurs créations, empêtrés qu'ils sont

1. Sylvie Chalaye, L'Afrique noire et son théatre au tournant du XX'e siècle, Rennes, PUR, 2001, p. 19. 
dans le vécu quotidien de leur société. Néanmoins, leur mimésis de la société exige aujourd'hui une réorientation, un renouvellement de la dramatisation du jeu politique, car l'Afrique multipartiste diffère indubitablement de celle des trois premières décennies des indépendances. L'expérience démocratique instaurée depuis 1990 a fait le lit de nouveaux maux que les dramaturges n'hésitent pas à pointer du doigt. En effet, confondant démocratie et ethnocratie, l'Afrique patauge désormais dans des considérations identitaires inquiétantes comme le souligne Caya Makhélé : «Les identités sont devenues tellement fortes, beaucoup plus fortes que l'idée de nation au point de faire de l'ethnie ou du groupe culturel la seule référence. $»^{2}$

Ce n'est pas Maurice Bandaman qui le contredira; lui dont le titre évocateur de la pièce $A u$ nom de la terre $e^{3}$ laisse apparaitre en toile de fond la question de l'identité, de la nationalité, voire du nationalisme qui secoue actuellement la société ivoirienne.

Depuis la fin de la décennie 1990, en effet, la société ivoirienne est agitée par le concept de l'ivoirité qui se voulait culturelle à sa création par Niangoran Porquet en 1973. Lié à la griotique, forme dramatique inspirée de l'art du griot, en réaction contre l'esthétique théâtrale occidentale, le concept était «le projet d'une recherche identitaire dont l'borizon est l'unité africaine $»^{4}$, ce qui est distinctif des Ivoiriens ne pouvant exister que dans un ensemble plus vaste (les griots appartenant à de nombreux peuples africains). Malheureusement, les machinations politiques vont le dévoyer pour en faire une théorie exclusionniste puisque des hommes politiques seront frappés d'ostracisme pour nationalité douteuse. L'atmosphère sociale se détériore, car une partie de la population se sent visée et frustrée par l'application biaisée du concept avec la catégorisation des citoyens. C'est cela que Bandaman met en scène dans son œuvre théâtrale.

Tributaire des festivités de fin d'année scolaire de l'École Normale William Ponty de Gorée au Sénégal, dans les années 1930, le théâtre ivoirien ${ }^{5}$ s'est émancipé et s'est forgé une identité à partir des années 1950 dans le cadre du Cercle Culturel Folklorique de Côte d'Ivoire (CCFCI) avec des auteurs comme Amon d'Aby, Coffi Gadeau, Bernard Dadié, dont les pièces visent l'éveil de conscience face à certains aspects des coutumes. Ce théâtre des mœurs inaugurait la réflexion de la société dans et par le théâtre. La désillusion postcoloniale renforcera ce lien avec la dramatisation du pouvoir notamment dans Les Voix dans le vent de Bernard Dadié ou La Termitière de Zadi Zaourou. En somme, ce théâtre se nourrissant des contingences socio-politiques du pays, se veut un regard sans complaisance de soi sur soi. Bandaman en perpétue le principe.

2. Ibid., p. 50 .

3. Maurice Bandaman, Au nom de la terre, Abidjan, EDUCI, 2000.

4. Ramsès Thiémélé, L'Ivoirité entre culture et politique, Paris, L'Harmattan, 2003, p. 132.

5. Lire de Koffi Kwahulé, Pour une critique du théatre ivoirien, Paris, L’Harmattan, 1996. 
Auteur, Maurice Bandaman est surtout reconnu pour sa verve romanesque dont Le Fils de-la-femme-mâle et La Bible et le fusil en constituent l'essence. Ses romans tirent leur substrat de l'alchimie de la littérature orale africaine et des nouvelles formes romanesques pour parvenir à une écriture postmoderne saupoudrée d'africanité avec l'emploi des techniques de l'oralité pour faire ressortir l'idéal de liberté et l'humanisme qui l'habitent. Soucieux du devenir de son peuple, il dénonce les périls qui le guettent comme avec $A u$ nom de la terre, pièce qui prend, selon lui, appui sur des faits s'étant déroulés quelque part en Côte d'Ivoire. Bien entendu, et dans une acception synecdochique, son pays est ce singulier qui dit le pluriel et l'universalité de la question identitaire. Comment le dramaturge dit-il une question qui soulève les passions? Quelle en est l'ampleur ? Pourquoi cette mise en scène de la question identitaire ? Tels sont les enjeux de cette étude.

\section{La théâtralisation de la question identitaire : entre réalité et fiction}

Pour Maurice Bandaman, la question identitaire avec son corollaire de nationalisme est de quintessence politique. En parler suppose alors la mise en scène du jeu politique. Pour y parvenir, son esthétique dramatique oscille entre le métathéâtre et l'omniprésence du symbolique.

\section{La mise en scène de la question identitaire ou l'esthétique du théatre dans le théâtre}

L'incipit de la pièce coïncide avec la didascalie initiale «Un jour. Place publique. Une foule assemblée autour d'un tribun». (p. 13) Au-delà des indications spatiotemporelles, celle-ci suggère une mise en place, expression d'une manifestation populaire : un meeting politique. Le message du tribun l'élucide :

«Frères et sœurs, l'heure a sonné ! La foule murmure. Oui, je dis que l'heure a sonné ! Notre heure! Pour retrouver notre honneur, notre dignité, notre liberté, c'est-à-dire nos droits. » (p. 13)

Le tribun annonce le thème du rassemblement. Il ressemble par ce fait au personnage de l'aboyeur du théâtre forain, faisant ainsi du meeting politique un spectacle théâtral. Ainsi, nous avons un acteur face à un public qui diffère de celui venu assister à la représentation. Subrepticement, Bandaman conçoit que les manifestations politiques sont comparables à des spectacles dramatiques, avec des acteurs face à un public de badauds qui réagit comme le ferait le public de la salle tel que le laissent entrevoir les didascalies relatives aux émotions et à l'attitude de la foule : "Applaudissements» (p. 13), "La foule burle de joie » (p. 14), «La foule exulte » (p. 14), «La foule en délire » (p. 15). La foule n'est pas insensible au spectacle offert par Le tribun : c'est la mise en abyme, cette représentation dans la représentation. 
Toutefois, ce métathéâtre supprime l'une des spécificités du dialogue théâtral, la double destination : «Le dialogue théâtral a toujours deux destinataires. L'auteur s'adresse au public en même temps que ses personnages se parlent entre eux. $\|^{6}$ Or ici, le discours du tribun n'est pas adressé à la salle mais à la foule venue l'écouter. Sa voix ne saurait donc être celle de l'auteur. Celui-ci prend ainsi ses distances avec les propos tenus par le tribun. N'étant pas ce dernier, il ne saurait être tenu pour responsable de ses propos. Il se prémunit contre tout relent identitaire dont il pourrait être taxé. Par conséquent, il pousse le lecteur-spectateur à la distanciation brechtienne, ce recul par rapport au spectacle nécessaire à l'exercice de l'esprit critique. Le destinataire additionnel du discours du tribun n'étant pas le public présent dans la salle de la représentation mais ses partisans. Le lecteur-spectateur comprend alors que le jeu politique se recouvre bien souvent du mensonge théâtral : il tire sur la vérité sans en être une.

\section{La représentation allégorique du nationalisme}

Semblable à une épigraphe, la didascalie initiale consacrée à la présentation des personnages de la pièce est précédée de la mention suivante : "Pièce inspirée de faits réels s'étant déroulés dans une région de la Côte d'Ivoire ». (p. 10) Cette mention fixe de façon indiscutable la réalité des faits et évènements présentés ainsi que le territoire de ces faits. Néanmoins, cet ancrage réaliste se trouve atténué par l'imprécision de la région et par l'emploi de l'adjectif épithète "inspiré », expression de la transfiguration et non de la transposition des faits. Il ne s'agit pas d'une pièce documentaire. Seulement la réalité s'incruste dans la fiction. Chez Bandaman, la fiction théâtrale est sous-jacente aux symboles théâtraux: le nanisme du candidat et l'escabeau. Pour la présentation du candidat, la didascalie précise : «Un nain escorté de jeunes filles. Il est si petit que pour se faire voir, il est obligé de monter sur un escabeau.» (p. 15) Le dramaturge dépasse le risible apparent pour faire du nain et de l'escabeau des emblèmes représentatifs du fondement du concept de nationalisme. Ils acquièrent une valeur allégorique manifeste.

Malgré sa ressemblance au brodequin des acteurs comiques antiques, l'escabeau surmonte son statut de simple accessoire de jeu pour dire le désir d'ascension qui anime le candidat. Il exprime sa volonté de prendre une revanche sur la nature qui l'a doté d'une morphologie peu avantageuse. En servant de support du paraittre au candidat porteur de l'idéologie nationaliste, l'escabeau se lit comme son but: décomplexer et rendre fier. L'escabeau impulse la régénérescence de cette dignité bafouée (frustration liée à la taille inférieure à la norme admise) et l'orgueil (dépassement psychologique de son handicap social) de s'affirmer aux yeux de tous. Le nationalisme promeut

6. Michel Pruner, L'Analyse du texte de théâtre, Paris, Armand Colin, 2008, p. 21. 
l'affirmation de soi, la reconnaissance de son droit à la différence. Il s'agit de lever le tabou sur son être afin de dire son appartenance à la communauté humaine.

Le candidat: [...] Je suis votre frère, nous avons le même sang, les mêmes ancêtres, nous parlons la même langue, pratiquons la même religion, adorons les mêmes fétiches. (p. 18)

L'identité est l'ensemble des valeurs civilisationnelles que l'on a en partage avec d'autres hommes comme stipulé par la répétition de "même ». L'escabeau se perçoit ainsi comme l'expression de la mêmeté du candidat. L'essence de l'être, c'est sa culture et non sa taille. Le candidat peut alors s'indigner quand il estime son identité mise à mal ou contestée: «Mais dites donc! Sur quelle terre sommes-mous? La sienne ou la nôtre ?» (p. 17) La fausse interrogation annonce le projet nationaliste: défendre son identité désignée par sa terre (la nôtre) signifiant l'idée de nation.

D'autre part, le nanisme du candidat symbolise de prime abord le complexe d'infériorité à l'origine d'un objet aidant à grandir. Le nationalisme s'appréhende alors comme l'admission de sa marginalisation, de son existence à la périphérie des autres. Plus qu'une réaction d'orgueil, il atteste le déficit social à combler par la proclamation de son identité, de sa spécificité. Aussi, étant donné que la population de nains est infime au sein de la société, on peut en déduire que le recours à un nain comme porte-voix du nationalisme implique la mise en minorité des nationalistes. Leur virulence tend à cacher leur insignifiance numérique. Dès lors, l'objet qu'est l'escabeau se mue en action de conquête. Il supplante le nain de sorte que celui-ci, loin de s'affirmer, s'aliène plutôt car ne pouvant être sans un appui. C'est cela le paradoxe nationaliste qui présage le tragique qu'il entretient.

\section{Le tragique de la question identitaire}

La question identitaire peut « provoquer des bouleversements énormes, susceptibles d'ébranler les valeurs ordinaires de l'humanisme. » ${ }^{7}$ C'est ce qui la rend tragique, comme est tragique tout ce qui fait perdre à l'homme son humanité.

\section{Le nationalisme ou le tragique de la victimisation de soi}

La thématisation du nationalisme par Le Candidat l'envisage comme une riposte à l'invasion des étrangers. C'est une doctrine de renaissance, de reconquête de la dignité perdue : "Vous m’avez sollicité pour que je défende votre honneur.» (p. 16) Il symbolise la contre-offensive contre l'agression

7. Sylvie Chalaye, op. cit., p. 50. 
étrangère, la persécution étrangère. Le nationalisme est d'essence paranoïde ; d'où le tragique : «Ils nous ont pris tous les postes et ce sont tous ces postes que nous devons reprendre.» (p. 19) L'exagération de la mainmise étrangère sur la société d'accueil sous-jacente à l'emploi du terme d'absolu «tous » dépasse le simple délire schizophrénique pour laisser poindre l'intolérance ; la formule impersonnelle "nous devons» préconisant une lutte sans concession contre l'étranger, ce responsable de tous les maux. Le nationalisme s'apparente ainsi à une schlague, expression de l'immodération dans la révolte. Or la violence conduit à la démesure qui, depuis le théâtre antique grec, est d'essence tragique.

Le tragique de la question identitaire résulte de la confrontation avec l'étranger auquel on entend s'imposer et imposer sa suprématie. Comme dans le théâtre grec antique dans lequel le tragique naissait de la révolte de l'homme contre le diktat des dieux dans un relent de quête libertaire et d'affirmation de la volonté humaine comme contre-pouvoir à la transcendance divine, le tragique du nationalisme découle de la révolte contre l'omniprésence étrangère qui implique un repli sur soi avec la proclamation de son identité en forme de défense et illustration de ses valeurs de civilisation: «Je suis votre frère, nous avons le même sang, les mêmes ancêtres, nous parlons la même langue, pratiquons la même religion, adorons les mêmes fétiches. » (p. 16) Dans son manifeste, le nationalisme part d'un bon sentiment. Seule sa mise en œuvre conduit au tragique.

Dans la pratique, le nationalisme s'inscrit dans la logique obscurantiste de la néantisation de l'autre, de la négation de son existence. On ne veut pas cohabiter avec lui mais l'écraser. On ne cherche pas à l'assimiler mais à le détruire, car celui-ci est perçu comme un indésirable, voire une non-personne, attesté par le mélange du registre familier et du langage oral qui fait de l'autre un simple référent affublé de termes dévalorisants : «cette ordure » (p. 16), «cette espèce d'andouille mal culottée» (p. 16), «cette boule de merde»(p.16), 《l'ordure» (p. 17), «l'étranger» (p. 17), «le voleur» (p. 17), «trafiquant» (p. 18), «fraudeur» (p. 18), « un faux-type » (p. 18), « un va-nu-pieds » (p. 18), etc. À l'instar des dieux de la tragédie antique qui « célèbrent la servitude éternelle de l'espèce humaine et empêchent son entrée dans l'histoire $»^{8}$ en se jouant du héros, les identitaires s'emploient à détruire tous ceux qu'ils jugent indignes de les côtoyer ; ils les rabaissent. Pour demeurer au firmament, on fait dégringoler l'autre. L'outrance et l'outrage des propos dramatisent le tragique nationaliste : l'autre n'est rien, donc je suis. Cette négation de l'autre suggère l'extrémisme dont le corollaire est le nihilisme qui s'affiche alors comme un des traits essentiels du tragique de l'enjeu identitaire.

8. Philippe Ivernel, "Pour une esthétique de la résistance», in Alternatives théâtrales, nº7-68, avril 2001, p. 15. 
En effet, le nationalisme porte en lui les germes de la violence, du chaos social vu l'emploi de formules péremptoires à valeur de sentences et de nature oxymorique comme «il faut lui donner une leçon! Une leçon politique, une leçon de démocratie » (p. 18) et «il n'y a plus d'entente à rechercher, il n'y a plus de consensus.» (p.19) L'antagonisme émerge et la négation implique l'excès semblable à la démesure à l'origine de la perte du héros tragique arcbouté sur ses convictions, ses certitudes. Mais contrairement à la tragédie grecque antique où seul le héros paie son aveuglement, chez Bandaman, c'est l'ensemble de la société qui est menacé par l'intransigeance nationaliste.

Le candidat: Même si le sucre ne se vante pas d'être sucré, sachez qu'en politique le sucre doit se mettre en valeur, rivaliser avec le sel, détruire le miel, renverser le miel, le salir...! (p. 18)

La portée injonctive d'infinitifs de sens négatif tels « détruire », « renverser » et « salir » en forme de gradation descendante dénote de la déchéance qui guette la société en proie au nationalisme exacerbé. Le tragique de l'avatar social surgit de cet appel à transformer «le miel » en fiel, et cela de façon délibérée et consciente.

Sur les 189 répliques que compte $A$ u nom de la terre, 147 (soit 77,77\%) reviennent aux nationalistes contre seulement 42 (soit 22,22\%) pour les modérés dont 5 (soit 11,9\%) pour Woblé, repenti. La prédominance des radicaux fait de la pièce une tragédie de la passion politique et non des passions comme chez les classiques. Conçue comme une pièce-paysage selon la classification de Michel Vinaver, l'œuvre peint les différentes facettes de la seule passion démesurée des nationalistes extrémistes. À l'instar des classiques, Bandaman procède à une redéfinition de la fatalité qui se veut non transcendantale mais consécutive à la submersion de la volonté humaine par la déraison politique. Il crée des personnages instigateurs qui n'agissent pas sur un évènement mais provoquent une situation. D'ailleurs la prédominance du présent de l'indicatif et l'appartenance de Bandaman au référent spatial évoqué proscrivent l'éloignement classique pour instaurer une proximité temporelle qui mue la tragédie de l'histoire chez les classiques en tragédie de l'actualité propice à une réflexion sur l'identité nationale comme projet politique. La responsabilité des politiciens est engagée dans l'actualité chaotique de leur société. Aussi, la finalité de la tragédie chez Bandaman rejoint celle des classiques. Pour lui aussi, et d'une certaine façon, « la tragédie renvoie (le politique) à l'exigeante réalité de sa liberté dans l'Histoire. »?

En réalité, c'est l'enjeu politique qui travestit le nationalisme et le rend démoniaque, machiavélique : «La politique c'est la guerre, c'est la mort !» (p. 18) Advienne que pourra si l'homme politique ne doit pas triompher. Le projet nationaliste se fait alors délibératif et prospectif à l'image de la destinée

9. Bruno Clément, La Tragédie classique, Paris, Seuil, 1999, p. 45. 
humaine que le héros tragique antique tente en vain de déjouer : "Ceux qui ne sont pas avec nous sont contre nous! Et ils ne méritent que trois choses : l'humiliation, l'exclusion, la mort ! (p. 19) Cette péroraison en forme de chute exprime la dérive tragique du nationalisme politique : l'appel au meurtre qui rappelle la notion de bouc-émissaire. L'étranger est le sacrifice expiatoire des ambitions politiques. En somme, le nationalisme n'est pas tragique en soi. Il le devient quand des hommes entendent, pour des raisons électoralistes, sanctionner la différence des origines. Autant deux volontés ne pouvaient coexister dans la tragédie grecque antique, autant différentes ethnies ne sauraient, selon le nationalisme politique, cohabiter harmonieusement. Le désir de pérennisation d'une culture devient la nécessité de préserver un espace de la supposée menace que représente l'autre.

\section{Le fonctionnement du tragique dans Au nom de la terre}

Il se matérialise dans l'ancrage réaliste à l'œuvre dans la pièce. L'onomastique des personnages y participe avec des personnages archétypaux. De nombreux noms sont porteurs de sens et prédestinent l'être et le faire du personnage, le plongeant dans une fatalité existentielle aveuglante. Le microcosme social d' $A u$ nom de la terre regorge de ces noms aux emplois codifiés et sémantiquement chargés. On y rencontre N'Datè, le «mauvais jumeau » (de N’Da: jumeau/ tè : mauvais, méchant), Woblé, le « serpent noir» (de wo : serpent/ blé: noir), WoOklé, le «venin de serpent» (de wo: serpent/ Oklé: venin) et Blatè, la "méchante femme» (de bla: femme/ tè : mauvais, méchant). Ces noms à consonance négative révèlent un univers où le mal règne, un monde de malfaisance. Aussi, il n'y a point de surprise quand on découvre qu'ils appartiennent tous à une confrérie de sorciers. L'évocation de cette société sécrète crainte en Afrique pour sa capacité de nuisance ne provient pas du folklore théâtral africain établi par les doctes en quête d'exotisme. Cette couleur locale métaphorise les leaders nationalistes, puisque tous ces personnages sont les adjuvants du candidat, leur frère.

Le dramaturge renvoie alors au venin, au poison de l'identité présentée comme un mal diffus, tapi dans l'ombre comme le sont les sorciers, ces êtres du monde irréel, irrationnel. Êtres de la nuit selon l'imagerie populaire, les sorciers sont perçus comme des mangeurs d'âme impitoyables. Par extension, est sorcier tout individu qui appelle au crime, au meurtre comme Blatè. L’intolérance identitaire conduit au crime. Le génocide rwandais de 1994 témoigne de cette triste vérité. Le dramaturge met en scène la chasse aux sorcières véhiculée par la crise identitaire. Que la femme, donneuse de vie, incite à l'ôter laisse perplexe et fait désespérer de l'espèce humaine dont le psychisme cache des profondeurs abyssales. Descartes a préjugé du bon sens des hommes en formulant «je pense donc je suis ». En réalité, cette sorcellerie identitaire, 
cette folie cache des ressentiments mal contenus. Blatè ne pardonne pas à N’Dakpa, dont elle réclame la mise à mort, de l'avoir éconduite au profit d'une autre femme. Son frère N'Datè lui reproche sa réussite sociale qui lui fait ombrage. À Woblé, il a ravi l'amour de Blatè à leur jeunesse. Dès lors, seules la jalousie, l'aigreur et la revanche commandent les horribles agissements des leaders nationalistes. Voilà le non-dit malsain de la crise identitaire; d'où la résistance de N'Dakpa qui atteste du fonctionnement du tragique par l'opposition des personnages.

Par son nom N'Dakpa, c'est-à-dire le «bon jumeau » (de N'Da: jumeau/ kpa: bon), ce personnage représente la raison, la sagesse, la modération. Il constitue l'îlot de bon sens qui résiste encore aux démons identitaires.

N’Dakpa : Je vous répète que je n'ai soutenu ni un étranger, ni un ennemi ni un inconnu. J'ai plutôt voté pour un frère, pour un homme qui me parlait d'amitié, de fraternité, d'entente, de partage, d'instruction, de santé ! Il parlait de l'avenir des jeunes. Et il n'insultait personne et ne maudissait personne. Et cet homme, je l'ai senti plus proche de moi. [...] Oui, j’ai préféré cet homme, son drapeau, ses couleurs, son discours au frère de sang, au frère d'ethnie, à son drapeau bariolé de sang, à ses couleurs aveuglantes. (p. 27)

La réplique enseigne que seul doit compter le faire et non l'être de l'individu. Il prône la démocratie au détriment de l'ethnocratie par le simple accomplissement de son devoir de citoyen. Une opposition idéologique s'installe en vue de mettre en exergue l'atmosphère délétère qui prévaut. N’Dakpa n'est donc ni un héros, ni un héraut. Il est un simple citoyen qui agit selon ses propres convictions. Il acquiert ainsi quelques traits du personnage ionesquien, notamment Bérenger de Rhinocéros. Dans une société atteinte de démence ethnocentriste, son choix citoyen en fait un franc-tireur de la libre pensée et insuffle à la pièce son civisme destiné à désiler les consciences aveugles et aveuglées.

\section{Au nom de la terre: une pièce rebelle}

Le drame chez le nationaliste jaillit de l'idée qu'il se fait de lui-même : il est tout ce qu'il y a de meilleur : "Votez pour moi, moi le beau, moi le juste, moi le généreux!» (p. 18) L’emploi emphatique de «moi » et des adjectifs valorisants «beau », "juste» et "généreux» dont se targue Le Candidat colporte la surestimation de soi pour ériger le nationalisme en idéologie du culte de la personnalité. Il se fait affabulation qui affaiblit sa portée.

\section{La démystification du discours identitaire}

Dans Au nom de la terre, Maurice Bandaman s'emploie à une démystification incidente du discours identitaire. La dynamique des relations entre politique et 
culture qui fonde le nationalisme repose sur la récupération et l'exploitation qui sont faites de la fibre nationaliste qui habite tout peuple. La pièce dramatise un nationalisme politique apparaissant comme un crime de lèse-culture, une atteinte à l'authenticité rayonnante de la culture défendue. Les éléments culturels sont dévoyés, désacralisés. Ainsi l'art divinatoire dont s'origine le respect pour des personnages comme Tirésias perd de sa substance au contact du politique : il est expressément demandé au devin Moussoublé (p. 38) d'accuser à tort N'Dakpa afin de justifier sa mise à mort. La culture couvre le déni de justice en versant dans la manipulation politique. Aux mains des politiques, la culture se révèle un instrument du mal, le terreau de la duplicité et de la fourberie. Quand la religion traditionnelle (p. 39) est convoquée à travers des signes ostentatoires tels les statues, les fétiches, les masques, elle est vidée de sa profondeur et de sa substance car les objets du culte sont profanés puisque mêlés à des squelettes humains et d'animaux ainsi qu'à des plumes diverses pour former un ensemble grotesque répulsif. Et lorsque l'adoration se fait avec du sang humain, la souillure de l'identité culturelle par les enjeux politiques jaillit. Le Candidat et ses lieutenants ont recours aux croyances anciennes à des fins maléfiques : éliminer un adversaire politique. L'image de la culture offerte par les nationalistes est peu flatteuse. Cela dessert la sincérité des tenants de la spécificité culturelle comme projet politique. Le dramaturge discrédite alors les leaders nationalistes en mettant en exergue leur imposture.

À cet effet, la double énonciation sert à démasquer le populisme pernicieux des discours identitaires contenu dans le mélange du registre lyrique et de la tonalité lyrique dont s'imprègnent les propos du candidat: "Cette boule de merde faite homme ose dire que je suis esclave, moi, votre frère, votre fils, propriétaire de la terre!» (p. 16) La gradation ascendante suggérée par l'emploi de «frère », « fils » et " propriétaire », grâce à l'intensité en crescendo, élucide la passion qui étreint le personnage tout en dévoilant son manque de lucidité. L'emphase et le point d'exclamation traduisent plus une indignation personnifiée qu'une quelconque colère née de l'humiliation subie par la collectivité. Le discours se fait démagogique avec l'exploitation malicieuse faite du rappel de la filiation. Celle-ci favorise la transformation du Moi en Nous destinée à rassembler et à créer un consensus autour du représentant de l'ethnie invitée et/ou incitée à laver l'affront subi par un individu. Le nationalisme politique dramatisé ici ignore la nation, car destiné à établir une connivence avec une ethnie.

Par ailleurs, la double énonciation démaquille le parti-pris du discours nationaliste à travers la modélisation discursive destinée à ternir l'image de l'étranger: "Un règne scélérat» (p. 16), « un règne brutal» (p. 16), "règne impie de ce bâtard d'étranger» (p. 16). Ces commentaires semblables à des slogans politiques expriment la prédominance de la subjectivité, de la partialité, de la haine qui discrédite le discours. Ce faisant, le dramaturge parvient à signaler au lecteur-spectateur l'hypocrisie du candidat qui se révèle à la fois juge 
et partie. Le procès de l'étranger dont se nourrit la question identitaire est un simulacre de procès, un procès biaisé. La véhémence du réquisitoire relevant de la stratégie de communication lors d'une campagne politique, Bandaman se fait ironique : il discrédite le candidat qui cherche à discréditer son adversaire par des attaques personnelles et déloyales.

En réalité, la double énonciation invalide les discours identitaires en leur enlevant toute valeur démonstrative. Ainsi, les propos outranciers tenus par l'étranger sont rapportés au discours indirect qui souffre de la possibilité de déformation des propos tenus. Ce qui est dit en devient alors peu crédible de sorte que le rapporteur lui-même voit son compte-rendu devenir contestable. Le discours indirect est la modalité discursive de la rumeur, parfois de la calomnie. Bandaman parvient ainsi à sous-entendre que les récriminations nationalistes peuvent être de simples affabulations et relever de la fertilité de l'imagination d'un individu. On peut en déduire que le nationalisme se nourrit quelquefois du mensonge. C'est ce qui le rend démagogique. La justesse du projet nationaliste est déformée par la passion des leaders comme l'indique cette didascalie relative à la tonalité du candidat: "Voix d'abord faible puis s'amplifiant progressivement.» (p. 16) Or la passion, selon le classicisme, doit se nourrir d'un peu de raison au risque de conduire au désastre. La passion immodérée étant déraison, le nationalisme devient contestable.

\section{La contestation du fanatisme nationaliste}

Le nationalisme tel que perçu et défendu par Blatè est jusqu'au-boutisme et obstination. Pour elle, l'échec est inenvisageable et le triomphe, vital.

Blatè : Arrêtez? (Pause.) Arrêtez? Mais arrêtez quoi ? Si nous arrêtons, nous mourrons. Nous avons jusqu'à la nuit de demain pour tuer N'Dakpa. Et nous devons aussi éliminer N'Dahou, cette petite morveuse qui a fait échouer notre plan. Nous ne pouvons arrêter, nous ne devons pas mourir. (p. 44)

Pour Blatè, renoncer à la fibre nationaliste équivaut à la mort. Aussi, elle s'appréhende comme un agent tragique, car faiseuse de la destinée des autres. N'a-t-elle pas scellé le sort de N'Dakpa et N'Dahou ? Le nationalisme se révèle ainsi comme une fatalité pour les sociétés qui l'expérimentent. Il conduit inexorablement à la mort du partisan qui faillit ou à celle des opposants. Le nationalisme est nécrophage. C'est pourquoi il est un mal dont il faut guérir l'ensemble de la société.

Pour Bandaman, le nationalisme est l'œuvre d'âmes insensibles sinon perdues. Woblé y souscrit, lui qui pourfend l'acharnement de son acolyte Blatè contre N'Dakpa et N'Dahou : «Maudite, sois-tu ! Femme au cœur de pierre ! » (p. 47) Seule l'absence de sentiment humain et/ou humaniste métaphorisée par «cœur de pierre » peut aveugler au point de ne pas s'apercevoir du mal que 
répand la politisation de la fierté culturelle. Et puisque le nationalisme politique s'avère une malédiction désignée par l'invective "maudite, sois-tu», un exorcisme s'impose pour marquer la contestation du fanatisme qui en émerge. Le désenvoûtement de la société victime du sort nationaliste s'impose. Ainsi, le repentir de Woblé s'accompagne d'une résolution : aider les hommes à survivre aux dérives nationalistes.

Woblé : Oui, nous mourrons tous les deux. Mais je ne te rejoindrai pas sans avoir guéri N'Dahou. Oui, il faut que je lui administre l'antidote du poison que tu lui as filé dans les entrailles. (p. 47)

La fatalité nationaliste n'est pas irrémissible.

L'un des antidotes au poison identitaire demeure l'amour du prochain, l'acceptation du droit à la différence qui ne signifie nullement négation de soi ou renonciation à son identité. D'ailleurs, l'éclat d'une identité ne peut mieux luire que parmi d'autres identités. Respecter la vie humaine, c'est faire abstraction des différences : "Arrêtez de haïr et acceptez d'aimer, acceptez d'aimer la vie, la beauté, l'amour, la liberté et... arrêtez. » (p. 42) L'impératif déploie à la fois un sermon et un code de bonne conduite dont la mise en œuvre prend forme avec la prise de conscience qui s'opère au sein même des nationalistes. Le deus ex machina se transforme en homo ex machina. En effet, le coup de théâtre provient de la révolte de Woblé contre lui-même et contre Blatè son alliée. Un tel revirement de situation signifie que les hommes ont le remède au mal qui ronge la société nationaliste. Le repentir de Woblé gage un nouvel ordre social.

Pour le dramaturge, en effet, le monde nationaliste doit faire place à un monde nouveau lavé des souillures de l'ordre ancien. Au moment où meurent Woblé et Blaté, N'Dahou met au monde un enfant en plein jour et sur la place publique comme marquer la fin de la longue nuit identitaire.

La Femme : Oui! Pousse! Pousse la vérité et la liberté en plein jour! Qu'est-ce que nous voyons là ? Un corps vert, un corps luisant d'amour et des bras pour embrasser l'amour, et des mains pour écrire la gaieté, et des pieds pour marcher, marcher sur de nouveaux sentiers, sur une terre nouvelle, une terre pure et purifiée ! Oui ! Pousse ! Pousse la vérité ! (p. 48)

Les encouragements de La Femme à l'endroit de N'Dahou se veulent en même temps une exhortation du peuple présent à cultiver un nouvel état d'esprit. La douleur de l'enfantement de N'Dahou signifie l'effort violent que la société doit faire sur elle-même pour parvenir à l'entente et panser ses plaies ouvertes par la crise vécue. Le corps luisant du nouveau-né symbolise la lumière qui fait fuir la nuit de l'extrémisme tandis que le corps vert traduit l'abondance à venir, la renaissance, le printemps d'une société pendant longtemps victime des préjugés destructeurs du repli identitaire fossoyé, torpillé par la malveillance des politiciens. 
Au nom de la terre a exclu le clivage dictature et opprimés en révolte. Au demeurant, la pièce tente d'attirer l'attention des hommes sur les dérives de l'engagement identitaire politisé. En opposant les jumeaux N'Dakpa et N'Datè, la pièce va au-delà des divisions et querelles familiales pour désigner la collectivité, les tensions internes qui secouent la société du fait de la fracture sociale inhérente à la question identitaire. Ce faisant, l'œuvre dramatique s'oriente vers le psychodrame. N'Dakpa et N'Datè incarnant cette investigation psychologique visant à analyser et soigner les conflits intérieurs, exorcisme indispensable à la disparition de la psychose de l'étranger à l'origine de la haine identitaire. Bandaman incite à la modération, au respect de l'autre. Et si cette pièce peut paraître engagée politiquement du fait de la dramatisation de l'identité politisée, il s'agit d'un engagement citoyen puisqu'en est exclu tout esprit de confrontation.

Aussi, Au nom de la terre campe des personnages sans étoffe, caricatures construites suivant des idées reçues, des clichés. Pour autant, l'œuvre ne perd ni pertinence ni force. Ce manque apparent de profondeur révèle l'immobilisme intellectuel dans lequel baigne le débat sur l'identité nationale ; un débat sclérosé, enrobé de parfum de réchauffé quand celle-ci est prise dans la houle des enjeux politiques comme le dramatise Bandaman. En réalité, la pièce met en évidence le ridicule des idées identitaires bien souvent orientées vers la stigmatisation de l'autre, aux dépens de tout esprit critique, de tout bon sens. D'une certaine façon, la pièce repose sur une esthétique de l'outrance ou de l'outrage aux politiciens nationalistes dont les partisans ont bien souvent du mal à tenir un discours emprunt de profondeur. Le stéréotype l'emporte sur la réalité des faits. L'enjeu de cette pièce réside dans le portrait des universaux des idéologies identitaires, très crisogènes. Et si à la fin de la pièce les identitaires se rebiffent ou se repentissent, ce n'est pas par naïveté chez le dramaturge. Il exprime simplement le vœu de voir les cœurs s'épancher, les consciences s'ouvrir sur un monde plus juste et moins violent. Il dit son envie de conjurer la violence d'où qu'elle vienne et quelle(s) qu'en soi(en)t la/les raison(s). La rébellion ivoirienne qui dure depuis 2002 confirme ses craintes et fait de sa pièce une prémonition. Il n'est pas un oiseau de mauvais augure encore moins un diseur de «malaventure ». Seulement l'identité porte en elle les germes de la destruction des sociétés qui ne la canalisent pas. Son message, en tant qu'humaniste, est de promouvoir un monde qui soit celui de deux amours : soi et l'autre. 


\section{Bibliographie}

BANDAMAN (Maurice), Au nom de la terre suivi de La Terre qui pleure, Abidjan, PUCI, «Écriture dramatique », 2000, p. 9-48.

BOA THIÉMÉLÉ (Ramsès L.), L'Ivoirité entre culture et politique, Paris, L'Harmattan, « Points de vue concrets », 2003.

CHALAYE (Sylvie), L'Afrique noire et son théâtre au tournant du XX' siècle, Rennes, PUR, « Plurial», 2001.

CHARBONNIER (Marie-Anne), Esthétique du théâtre moderne, Paris, Armand, Colin/Masson, «Synthèse », série « Lettres », 1998.

CLÉMENT (Bruno), La Tragédie classique, Paris, Seuil, « Mémo », 1999.

COLLECTIF, Alternatives théatrales: Rwanda 94. Le théâtre face au génocide. Groupov, récit d'une création, Bruxelles, nº7-68, avril 2001.

COUPRIE (Alain), Le Théatre: texte, dramaturgie, histoire, Paris, Armand Colin, 2009.

DIOP (Samba), Discours nationaliste et identité ethnique à travers le roman sénégalais, Paris, L'Harmattan, 2003.

KWAHULÉ (Koffi), Pour une critique du théatre ivoirien, Paris, L'Harmattan, «Images plurielles », 1996.

PRUNER (Michel), L'Analyse du texte de théâtre, Paris, Armand Colin, 2005, 2008.

RICCEUR (Paul), Soi-même comme un autre, Paris, Seuil, « Points Essais », 1990.

SCHÉRER (Jacques), Littératures francophone d'Afrique noire, Aix-en Provence, Compagnie des éditions de la Lesse, Edisud, «Les Écritures du sud», 2006. 